\title{
The Anti-inflammatory Effect of Dexmedetomidine Administration on Patients Undergoing Intestinal Surgery: A Randomized Study
}

\author{
Rushuang Chen ${ }^{1} \cdot$ Zhenming Kang $^{1} \cdot$ Yaduan Wang ${ }^{1} \cdot$ Jie Zhao $^{1} \cdot$ Shunyuan Li ${ }^{1}$
}

Accepted: 8 October 2021 / Published online: 9 November 2021

(c) The Author(s) 2021

\begin{abstract}
Background and Objective Dexmedetomidine is a highly selective a2-adrenergic receptor agonist with sedative, analgesic, anti-sympathetic and stress-reducing effects. It has been widely used as an adjunct for general anesthesia of multiple surgeries. However, the relationship between the utilization of dexmedetomidine in intestinal surgery and the postoperative inflammatory response of patients remains unclear.

Methods A randomized, controlled, single-blinded clinical trial was performed. Eighty-six patients assigned for intestinal surgery were recruited and were randomly divided into two groups (dexmedetomidine group, $n=40$; control group, $n=40$ ) [six participants were excluded due to multiple reasons, such as allergy and drug use history]. The clinical characteristics and physiological outcomes of participants who received different treatments (dexmedetomidine and $0.9 \%$ sodium chloride) were collected and analyzed. Blood samples of the two groups were collected before administration (T0), 10 min after pumping dexmedetomidine/saline solution (T1), immediately after the operation started (T2), $30 \mathrm{~min}$ after the operation started (T3), and immediately after the operation ended (T4). Enzyme-linked immunosorbent assay (ELISA) was performed to evaluate the proinflammatory factors.

Results Intravenous injection of dexmedetomidine before intestinal surgery decreased a variety of circulating proinflammatory factors. Dexmedetomidine alleviated the stress response and promoted the recovery of cognitive ability among patients undergoing intestinal surgery.

Conclusion Dexmedetomidine administration in patients undergoing intestinal surgery inhibited the surgery-induced inflammatory reactions.
\end{abstract}

\section{Key Points}

Intravenous injection of dexmedetomidine before intestinal surgery reduces multiple circulating proinflammatory factors in patients' serum.

Dexmedetomidine alleviated the stress response and promoted the recovery of cognitive ability among patients undergoing intestinal surgery.
Zhenming Kang

kzm991041@163.com

Shunyuan Li

1i767679@sina.com

1 Department of Anesthesiology, Quanzhou First Hospital Affiliated to Fujian Medical University, Quanzhou 362000, Fujian, China

\section{Introduction}

The intestine is the organ with the largest surface area in the human body [1]. The intestinal mucosa damaged by surgery induces the production of a variety of proinflammatory factors, which leads to the activation of inflammatory cells and the release of multiple inflammatory mediators [2]. The inflammatory reaction caused by intestinal surgery can inhibit intestinal bacteria from invading peripheral blood and distance organs through the damaged intestinal mucosa [3]; however, excessive intestinal inflammation leads to damage and atrophy of intestinal mucosa, and thus decreases its barrier function [4, 5]. A large number of proinflammatory factors released by intestinal tissues can cause systemic inflammatory reactions, resulting in fever, peripheral blood leukocyte alteration, and impaired function of organs such as liver and kidney [6-8]. Therefore, controlling the intraoperative and postoperative inflammatory response of patients with bowel surgery and reducing the secretion of inflammatory factors 
are of great significance for improving clinical efficacy and prognosis, and prolonging their survival time.

Dexmedetomidine is a highly selective a2-adrenergic receptor agonist with sedative, analgesic, anti-sympathetic, and stress-reducing effects $[9,10]$. It has been widely used in clinic because it has no significant respiratory depression effect $[11,12]$. It has been reported that dexmedetomidine can function as a general anesthetized adjunct and reduce the amount of anesthetic drugs, thus maintaining hemodynamic stability and reducing patients' stress response during surgery [13]. The application of dexmedetomidine in cardiovascular surgery can also reduce the degree of restlessness and the incidence of hypertension in elderly hypertensive patients [14]. The application of dexmedetomidine infusion during laparoscopic surgery improves the cognitive function of patients and reduces the incidence of early postoperative cognitive dysfunction [15]. More importantly, in a craniotomy for the treatment of ischemic brain injury, dexmedetomidine treatment effectively reduces the content of various inflammation-related factors in patients' serum, including tumor necrosis factor (TNF)- $a$, interleukin (IL)-6, S100 calcium-binding protein- $\beta$ (S100- $\beta$ ), superoxide dismutase (SOD) and cortisol [16]. Utilization of low-dose dexmedetomidine effectively accelerates the intestinal function recovery post-surgery [15]; however, the relationship between the use of dexmedetomidine in intestinal surgery and the postoperative inflammatory response of patients remains unclear.

In this study, we mainly investigated the inhibitory effect of dexmedetomidine on the systemic inflammation caused by intestinal surgery, and demonstrated that preoperative intravenous injection of dexmedetomidine reduced the incidence of inflammation. We believe that our research provides a theoretical basis for the clinical application of dexmedetomidine and helps to improve the prognosis and life quality of patients with intestinal surgery.

\section{Methods}

\subsection{Study Design}

To investigate the correlation between the application of dexmedetomidine and the physiological outcomes of patients with intestinal surgery, a randomized, controlled, and single-blinded clinical trial was performed in this study. We evaluated the anti-inflammatory effects of dexmedetomidine on patients undergoing intestinal surgery from 2019 to 2020, and aimed to recruit patients arranged for intestinal surgeries. Patients who participated in our clinical trial were from Quanzhou First Hospital Affiliated to Fujian Medical University. This study was approved by the Ethics Committee of Quanzhou First Hospital Affiliated to Fujian Medical University (QZDIYY/2019/28), and followed the Declaration of Helsinki ethical principles for medical research involving human subjects.

\subsection{Participants}

Patients who met the following criteria were recruited in this clinical trial: (1) over 18 years of age; (2) meeting the American Society of Anesthesiologists (ASA) score I-II; (3) diagnosed by computed tomography (CT) and indicated for surgery; (4) no history of sedation and analgesics; (5) no contraindications for gastrointestinal surgery; (6) no dexmedetomidine allergies; and (7) signed informed consent and participated voluntarily. However, patients with the following situations were excluded from the trial: (1) combined respiratory and metabolic diseases; (2) combined endocrine and immune diseases; (3) stomach, liver and kidney dysfunction before surgery; (4) large-scale use of sedative drugs for a long period of time; (5) mental illness; and (6) disagree or were unwilling to cooperate with the investigator. The study was approved by the Ethics Committee of Quanzhou First Hospital Affiliated to Fujian Medical University.

A total of 86 patients meeting the above criteria were recruited and assessed for our study. Participants were randomly divided into two groups (dexmedetomidine group and control group) at a ratio of 1:1. Random allocation sequence was generated by a colleague who did not participate in this research. Among all patients, six participants were excluded due to multiple reasons such as allergy and drug use history; data were collected from the remaining 80 participants. Patients in the control group received an intravenous infusion of $0.9 \%$ saline $10 \mathrm{~min}$ before the induction of anesthesia, while participants in the dexmedetomidine group received an intravenous infusion of dexmedetomidine $10 \mathrm{~min}$ before the induction of anesthesia.

\subsection{Interventions}

A standardized anesthesia management protocol was applied among the groups. All patients were fasted for $12 \mathrm{~h}$ and deprived of water for $3 \mathrm{~h}$. After entering the operating room, patients were connected to an automated system (IntelliVue MP50, Philips, Amsterdam, The Netherlands). Electrocardiogram (ECG), pulse oxygen saturation $\left(\mathrm{SpO}_{2}\right)$, heart rate (HR), blood pressure (BP), and bispectral index (BIS; Aspect A-2000, Aspect Medical Systems Inc., Newton, MA, USA) were routinely monitored. 
Routine preoperative injection Diazepam $(10 \mathrm{mg})$ and atropine $(0.5 \mathrm{mg})$ were injected through the right internal jugular vein in hydroxyethyl starch 200/0.5 and sodium chloride injection at a rate of $6-8 \mathrm{ml} \mathrm{kg}^{-1} \mathrm{~h}^{-1}$. In the meantime, a left radial artery puncture was performed to monitor the invasive arterial pressure. Ten minutes before the induction of anesthesia, a loading dose of dexmedetomidine $1 \mu \mathrm{g} / \mathrm{kg}$ was injected by intravenous pump to the intervention group. Intravenous infusion of dexmedetomidine was then kept at a rate of $0.4 \mu \mathrm{g} \mathrm{kg}^{-1} \mathrm{~h}^{-1}$ until the end of operation. The same dose of saline was applied to the control group.

Anesthesia induction Midazolam $0.05 \mathrm{mg} / \mathrm{kg}$, medium/long chain propofol $1.0-2.0 \mathrm{mg} / \mathrm{kg}$, cisatracurium $0.10-0.15 \mathrm{mg} /$ $\mathrm{kg}$, and sufentanil citrate $0.3-0.4 \mu \mathrm{g} / \mathrm{kg}$ were injected intravenously. Patients were given mechanical ventilation with inhalation oxygen concentration of $60 \%$, tidal volume of $6-8 \mathrm{~mL} / \mathrm{kg}$, ventilation frequency of 10 times $/ \mathrm{min}$, inspiratory/expiratory ratio of $1: 2$, and oxygen flow rate of $2.2 \mathrm{~L} / \mathrm{min}$. PetCO $\mathrm{C}_{2}$ was maintained at $35-40 \mathrm{mmHg}(1$ $\mathrm{mmHg}=0.133 \mathrm{kPa}$ ).

Intraoperative maintenance Remifentanil 0.1$0.2 \mu \mathrm{g} \mathrm{kg}^{-1} \mathrm{~min}^{-1}$ and propofol $3-5 \mathrm{mg} \mathrm{kg}^{-1} \mathrm{H}^{-1}$ were infused intravenously and muscle relaxants were administered intermittently. During the operation, hemodynamic stability was maintained by adjusting the pump rate of remifentanil and propofol, and BIS values were maintained at 45-55. During the operation, lactate Ringer's liquid was used to supplement physiological requirement, and hydroxyethyl starch 200/0.5 sodium chloride was injected to supplement blood loss. During anesthesia, the dosage was adjusted according to the changes in HR, BP, and other indicators. Vital signs were closely monitored and kept stable.

Postoperative management After the operation, when patients had recovered completely, the tracheal tube was pulled out and patients were sent to the anesthesia recovery room for patient-controlled intravenous analgesia. The analgesic solution formula dezocine $25 \mathrm{mg}$ and tropisetron hydrochloride $10 \mathrm{mg}$ was diluted to $100 \mathrm{~mL}$ with $0.9 \%$ normal saline for injection. The loading dose was $5 \mathrm{~mL}$, background dose was $2 \mathrm{~mL} / \mathrm{h}$, additional dose was $1 \mathrm{~mL} /$ time, locking time was $10 \mathrm{~min}$, and analgesia time was $48 \mathrm{~h}$.

\subsection{Data Collection}

Two millimeters of blood was collected from the left radial artery of patients before administration (T0), 10 min after pumping dexmedetomidine/saline solution (T1), immediately after the operation started (T2), 30 min after the operation started (T3), and immediately after the operation ended (T4). The serum was separate by centrifugation and store at $-20{ }^{\circ} \mathrm{C}$ for further experiment. Enzyme-linked immunosorbent assay (ELISA) kits (R\&D Systems, Minneapolis, MN, USA) were used to evaluate the circulating cytokines and proteins, including IL-6, TNFa, IL-8, and IL-10, while turbidimetry assay was used to evaluate the $\mathrm{C}$-reactive protein (CRP) levels. The recovery time of spontaneous breathing, the time of tracheal tube removal, and the occurrence of postoperative agitation in the two groups of patients were recorded immediately after the operation. Mini-Mental State Examination (MMSE) was used to demonstrate the cognitive function of patients in each group before administration and 2, 6, 12 and $24 \mathrm{~h}$ after operation [17]. An MMSE score lower than 27 was considered as the incidence of postoperative cognitive dysfunction.

\subsection{Statistical Analysis}

To detect a difference of $\geq 20 \%$ in the cumulative number of inflammatory factors after operation, a sample size of 39 per group was required to have a study power of $80 \%(\alpha=0.05$, $\beta=0.2$ ), i.e. a total of 78 patients. Assuming a dropout rate of $10 \%$, a total of 86 patients were initially recruited for adequate data collection.

All data were obtained from three independently repeated measurements. The categorized variables are shown as frequency or percentage, while mean and standard deviation are utilized to demonstrate the continuous variables, all of which followed normal distribution. The Chi-square test (or Fisher's exact test) was used to compare the classification variables. One-way analysis of variance (ANOVA) and Tukey's post hoc test were used to analyze the continuous variables and a contingency table Chi-square test was acquired for the comparison of outcomes among different groups. SAS statistical software version 17.0 (SAS Institute Inc., Cary, NC, USA) was purchased for the statistical analysis in this study.

\section{Results}

\subsection{Study Procedure}

As shown in Fig. 1, a total of 86 patients meeting the above criteria were recruited and assessed for our study. Participants were randomly divided into two groups (dexmedetomidine group and control group). Participants in the dexmedetomidine group received intravenous dexmedetomidine 10 min before the induction of anesthesia.

\subsection{Basic Characteristics of the Participants}

The basic clinical characteristics of all participants were collected and are shown in Table 1. The demographic parameters collected from patients in the two groups included age, 
sex, body mass index (BMI), basic diseases, preoperative indicators, and duration of surgery and anesthesia. As shown in Table 1, there were no significant differences in any of the above characteristics between participants in these two groups at the beginning of our research $(p>0.05)$.

\subsection{Dexmedetomidine Treatment Suppressed Interleukin (IL)-6, IL-8, IL-10, Tumor Necrosis Factor- $\alpha$ and C-Reactive Protein Levels in Patients with Intestinal Surgery}

Inflammation-related factors of patients at different time points in these two groups are shown in Fig. 2. ELISA assay was used to evaluate the serum cytokines, including IL-6 (Fig. 2a), IL-8 (Fig. 2b), IL-10 (Fig. 2c), and TNFa (Fig. 2d) at T0-T4. Compared with the control group, IL-6 levels were lower at T2-T4, IL-8 levels were lower at T3-T4, IL-10 levels were higher at T2-T4, and TNFa was lower at $\mathrm{T} 2-\mathrm{T} 4(p<0.05)$. These differences suggested that dexmedetomidine infusion before anesthesia inhibited the systemic inflammation induced by intestinal surgery. The circulating CRP level of each patient was estimated by turbidimetry assay. Compared with the control group, the CRP levels of patients in the dexmedetomidine group were significantly lower at T2-T4 $(p<0.05)$ [Fig. 2e]. Therefore, treatment with dexmedetomidine before anesthesia significantly inhibited systemic inflammation during and post intestinal surgery.

Fig. 1 Flow diagram of the study. DEX dexmedetomidine

\subsection{Dexmedetomidine Treatment Reduced the Respiratory Recovery Time, Extubating Time, and Postoperative Agitation in Patients with Intestinal Surgery}

The recovery time of patients' spontaneous breathing, time of tracheal tube removal, and occurrence of postoperative agitation of participants were recorded and are shown in Table 2. Furthermore, there were statistical differences between the respiratory recovery time $(p=0.026)$, extubation time $(p=0.043)$, and postoperative agitation ( $p=0.009)$. Therefore, intravenous infusion of dexmedetomidine $10 \mathrm{~min}$ before anesthesia induction could not only improve the anesthesia effect but also promote the recovery of patients after anesthesia.

\subsection{Dexmedetomidine Treatment Increased the Mini-Mental State Examination in Patients Post Intestinal Surgery}

The MMSE was performed before administration and 2, 6,12 , and $24 \mathrm{~h}$ after surgery. As shown in Table 3, the MMSE scores of the dexmedetomidine group increased significantly at $2 \mathrm{~h}(p=0.032), 6 \mathrm{~h}(p=0.008), 12 \mathrm{~h}$ $(p=0.029)$, and $24 \mathrm{~h}(p=0.018)$ post-surgery compared with the control group. Therefore, intravenous infusion of dexmedetomidine 10 min before anesthesia induction

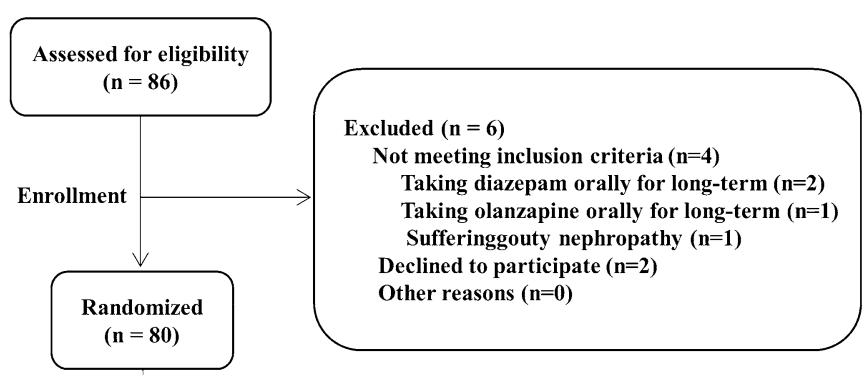

Allocation

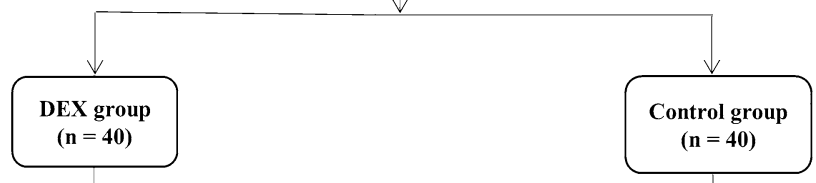

Follow-up

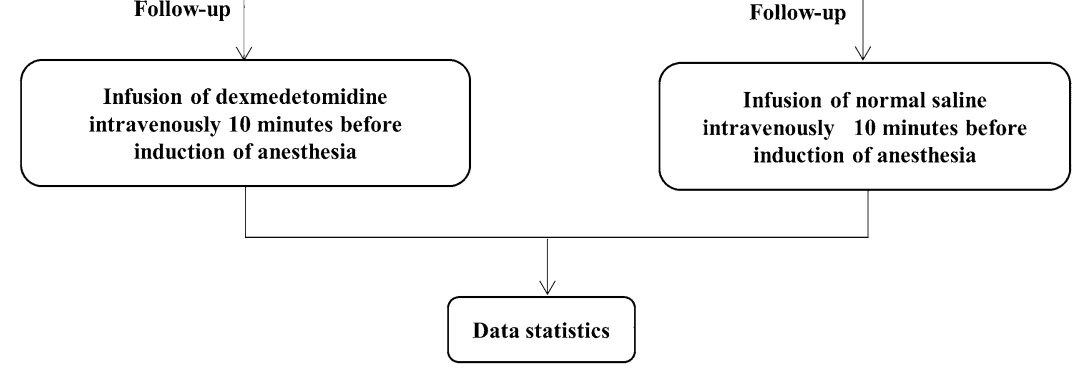


Table 1 Comparison of the two groups of patients using basic information

\begin{tabular}{llll}
\hline Items/groups & DEX group $[n=40]$ & Control group $[n=40]$ & $p$ value \\
\hline Age, years & $50.76 \pm 8.32$ & $51.07 \pm 9.43$ & 0.816 \\
Sex $[n(\%)]$ & & $21(52.5)$ & 0.329 \\
Male & $23(57.5)$ & $19(47.5)$ & \\
Female & $17(42.5)$ & $26.87 \pm 5.36$ & \\
Body mass index, $\mathrm{kg} / \mathrm{m}^{2}$ & $26.69 \pm 5.08$ & & 0.403 \\
Coexisting diseases $[n(\%)]$ & & $9(22.5)$ & \\
Hypertension & $7(17.5)$ & $6(15.0)$ & \\
Diabetes & $5(12.5)$ & $15(37.5)$ & 0.678 \\
Dyslipidemia & $13(32.5)$ & & 0.314 \\
Preoperative indicators & & $77 \pm 9$ & 0.509 \\
Heart rate, number/min & $76 \pm 11$ & $6.51 \pm 1.19$ & 0.215 \\
WBC counts, $10^{9} / \mathrm{L}$ & $6.23 \pm 1.45$ & $9.09 \pm 2.44$ & 0.623 \\
Neutrophils, $10^{9} / \mathrm{L}$ & $9.03 \pm 2.35$ & $244.87 \pm 51.67$ & 0.136 \\
Platelet count, $10^{9} / \mathrm{L}$ & $250.17 \pm 44.86$ & $96.18 \pm 6.07$ & 0.350 \\
SpO ${ }_{2}, \%$ & $96.43 \pm 5.16$ & $214.4 \pm 24.7$ & \\
Duration of surgery, min & $207.5 \pm 19.4$ & $265.2 \pm 30.5$ & \\
Duration of anesthesia, min & $260.8 \pm 26.3$ & & \\
\hline
\end{tabular}

Data are expressed as mean \pm SD

Participants in the DEX group received intravenous DEX 10 min before the induction of anesthesia $W B C$ white blood cell, $\mathrm{SpO}_{2}$ pulse oxygen saturation, $D E X$ dexmedetomidine, $S D$ standard deviation
A

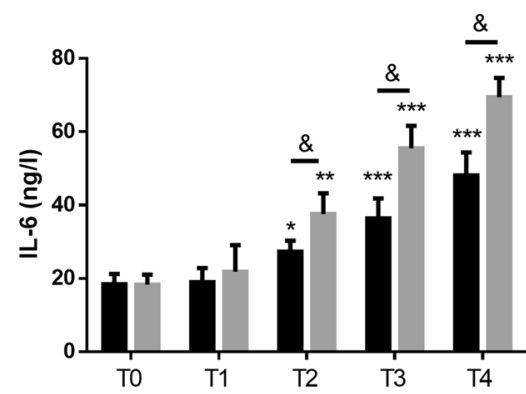

D

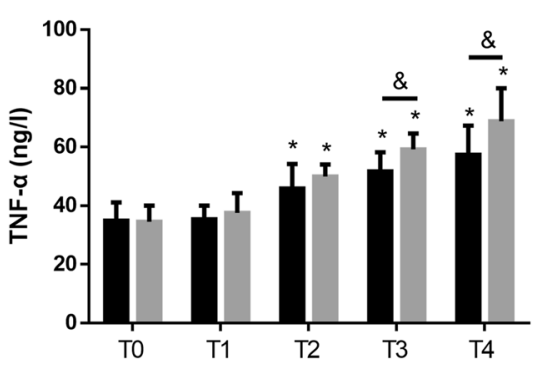

B

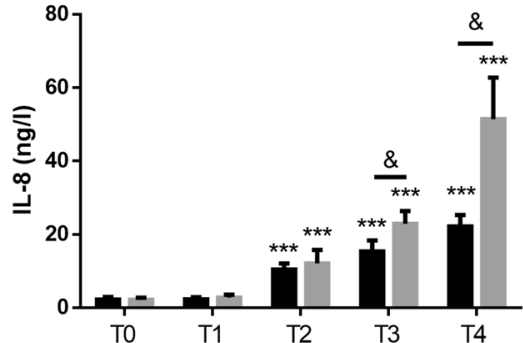

$\mathrm{E}$

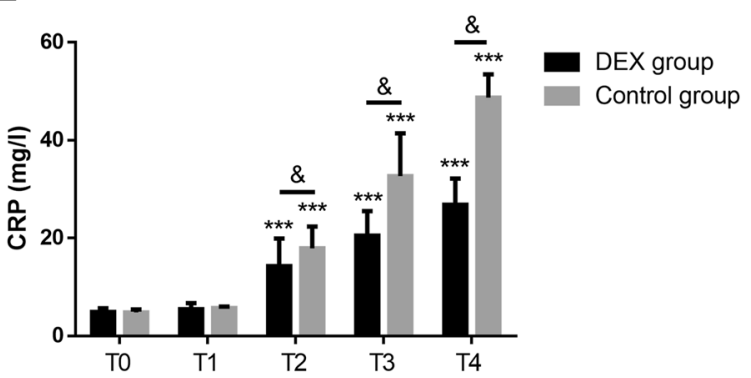

Fig. 2 Effect of dexmedetomidine administration on inflammation in patients undergoing intestinal surgery. Comparison of the value of a IL-6, b IL-8, $\mathbf{c}$ IL-10, d TNFa, and e CRP between the two groups of patients. Data are expressed as mean \pm SD. $* p<0.05$, $* * p<0.01$, and $* * * p<0.001$ compared with T0. Data in bold indicate significant differences between the two groups. $I L$ interleukin, $T N F$ tumor necrosis factor, $C R P$ C-reactive protein, $S D$ standard deviation, $D E X$ dexmedetomidine 
Table 2 Comparison of the value of respiratory recovery time, extubation time, postoperative agitation, and remifentanil consumption between the two groups of patients

\begin{tabular}{lccc}
\hline Items/groups & DEX group $[n=40]$ & Control group $[n=40]$ & $p$ value \\
\hline Respiratory recovery time, min & $6.54 \pm 2.06$ & $13.57 \pm 4.36$ & $\mathbf{0 . 0 2 6}$ \\
Extubation time, min & $13.48 \pm 4.78$ & $19.71 \pm 3.82$ & $\mathbf{0 . 0 4 3}$ \\
Postoperative agitation, $\%$ & 10 & 50 & $\mathbf{0 . 0 0 9}$ \\
Consumption of remifentanil intraop- & $343.7 \pm 78.3$ & $475.6 \pm 79.2$ & $\mathbf{0 . 0 0 2}$ \\
$\quad$ eratively, $\mu \mathrm{g}$ & & & \\
\hline
\end{tabular}

Data in bold indicate the significant differences between the two groups

$D E X$ dexmedetomidine

${ }^{\mathrm{a} C}$ Compared with the control group, $p<0.05$

\begin{tabular}{lllll}
\hline Index & Timepoints & DEX group $[n=40]$ & Control group $[n=40]$ & $p$ value \\
\hline MMSE & T0 & $27.53 \pm 6.45$ & $27.79 \pm 5.16$ & 0.445 \\
& $2 \mathrm{~h}$ & $24.15 \pm 7.53^{* * *}$ & $23.35 \pm 7.43^{* * *}$ & $\mathbf{0 . 0 3 2}$ \\
& $6 \mathrm{~h}$ & $25.36 \pm 4.62^{* *}$ & $23.89 \pm 6.14 * * *$ & $\mathbf{0 . 0 0 8}$ \\
& $12 \mathrm{~h}$ & $26.93 \pm 5.72^{*}$ & $24.76 \pm 6.34^{* *}$ & $\mathbf{0 . 0 2 9}$ \\
& $24 \mathrm{~h}$ & $27.47 \pm 5.35$ & $26.09 \pm 7.75^{*}$ & $\mathbf{0 . 0 1 8}$ \\
\hline
\end{tabular}

MMSE Mini-Mental State Examination, SD standard deviation, $D E X$ dexmedetomidine

Data are expressed as mean $\pm \mathrm{SD}$

${ }^{*} p<0.05, * * p<0.01, * * * p<0.001$ compared with $\mathrm{T} 0$

The $p$-value indicates the significant differences between the two groups; data in bold indicate the significant differences between the two groups effectively promoted the recovery of patients' postoperative cognitive ability.

\subsection{Dexmedetomidine Treatment Reduced the Adverse Reactions in Patients Post Intestinal Surgery}

Comparison of the incident of adverse reactions, including postoperative nausea and vomiting (PONV), shiver, respiratory depression, dizziness, and bradycardia, between the two groups of patients is shown in Table 4. Dexmedetomidine administration significantly decreased the incidence of PONV ( $p=0.008)$, shiver $(p=0.011)$, and dizziness $(p=0.013)$, suggesting that intravenous infusion of dexmedetomidine could effectively reduce the adverse reactions caused by anesthesia.

\section{Discussion}

Generally, multiple factors, including anesthesia, intraoperative bed rest, surgery, and carbon dioxide stimulation, can affect patients undergoing intestinal surgery, resulting in weakening or even disappearing of gastrointestinal motility and increasing difficulty in the recovery of gastrointestinal function [18]. The surgery will not only cause the patient to lose a large amount of fluid in the intestinal cavity but also
Table 4 Comparison of the incident of adverse reactions between the two groups of patients

\begin{tabular}{llll}
\hline Index & $\begin{array}{l}\text { DEX group } \\
{[n=40]}\end{array}$ & $\begin{array}{l}\text { Control group } \\
{[n=40]}\end{array}$ & $p$ value \\
\hline Adverse reactions & & & \\
PONV & $1(2.5)$ & $6(15)$ & $\mathbf{0 . 0 0 8}$ \\
Shiver & $2(5)$ & $12(30)$ & $\mathbf{0 . 0 1 1}$ \\
Respiratory Depression & $0(0)$ & $4(10)$ & NA \\
Dizziness & $3(7.5)$ & $10(25)$ & $\mathbf{0 . 0 1 3}$ \\
Bradycardia & $0(0)$ & $5(12.5)$ & NA \\
\hline
\end{tabular}

Data are expressed as $n(\%)$

Data in bold indicate the significant differences between the two groups

$P O N V$ postoperative nausea and vomiting, DEX dexmedetomidine, $N A$ not available

increase the patient's intestinal and intra-abdominal pressure, resulting in clinical symptoms such as abdominal pain, bloating, and intestinal paralysis, which affects the treatment effect $[19,20]$. Abdominal infection is one of the most common intraoperative and postoperative complications [21]. The main reasons include improper aseptic operation during the operation, damaged organs, and perforation or anastomotic rupture [22], which are also significant reasons why intestinal surgery can easily cause inflammation. In addition, during cardiopulmonary bypass (CPB) surgery, various 
stimulating factors often cause a strong stress response in the human body, among which stress inflammatory response is very common in patients undergoing laparotomy and thoracotomy. The severe inflammatory reaction caused by intestinal surgery may lead to cell degeneration, necrosis, or abnormal metabolic function in the wound area [23]. Mechanical obstruction and compression caused by a large number of inflammatory exudates can seriously interfere with wound healing after surgery [24, 25]. In addition, the inflammation induced by surgery may lead to fever and changes in circulating immune cells, and eventually lead to different degrees of degeneration, necrosis, and dysfunction of the parenchymal cells of the heart, liver, kidney, and other organs [26]. Therefore, inhibiting the inflammatory response and reducing the level of inflammatory response in patients after bowel surgery are of great significance to improve the clinical efficacy and prognosis of patients. Furthermore, it is also of great significance for prolonging the survival time of patients undergoing intestinal surgery.

In this study, we mainly investigated the relationship between the application of dexmedetomidine and the outcomes of intestinal surgery patients. We showed that dexmedetomidine could effectively reduce the level of inflammatory factors circulating in patients during surgery and inhibit the inflammation caused by surgery. In addition, we also demonstrated that intravenous infusion of dexmedetomidine before anesthesia could improve the anesthetic effect and reduce the adverse reactions caused by anesthetic drugs. At the same time, dexmedetomidine could also promote the recovery time and speed of consciousness recovery after intestinal surgery. We believed that our research provided a new theoretical basis for the application of dexmedetomidine in bowel surgery.

Dexmedetomidine is a highly selective adrenergic a 2 receptor (a2AR) agonist; its ratio of binding to a $2 \mathrm{AR}$ and a 1-adrenergic receptor (a1AR) is 1600:1 [27, 28]. The affinity of dexmedetomidine with a2AR is several times that of clonidine [29], and its efficacy is precise and dosedependent. Dexmedetomidine has definite anti-anxiety, sedation, and analgesic effects. Previous studies have shown that patients treated with dexmedetomidine before surgery ended up with significantly higher sedation scores than the control group [30]. Another study showed that even if the dose of dexmedetomidine reached multiple times the recommended dose, the patient's pulse, blood oxygen saturation, and arterial blood carbon dioxide partial pressure was still maintained within the normal range under the condition of inhaled air [31]. Thus, dexmedetomidine is widely used as a preoperative medication and its effect can continue until the induction period. Dexmedetomidine can be used in conjunction with other inducing drugs to deepen the depth of anesthesia and weaken the cardiovascular response, thereby reducing irritation, severe response, and difficult intubation operations, including double-lumen bronchial intubation, obstructive tube intubation, and in the chest cannula used in surgery [32]. In addition, dexmedetomidine has the effect of strengthening vagal tone and reducing sympathetic tone, which allows the application of dexmedetomidine to reduce HR and prevent surgery- and stress-induced hypertension [33]. Accumulating evidence has demonstrated that dexmedetomidine can stimulate central and peripheral receptors, reduce sympathetic nerve activity and plasma catecholamine concentration, and reduce perioperative stress response [10]. Its ability to reduce sympathetic tension and indirectly increase parasympathetic tension is of great importance to reduce the occurrence of inflammation, inhibit the release of inflammatory factors, reduce cell apoptosis, and reduce the occurrence of sepsis. Since the relationship between the use of dexmedetomidine in intestinal surgery and the postoperative inflammatory response of patients is still unclear, our research may provide a new perspective on the clinical application of dexmedetomidine.

However, there were still some shortcomings in our research. Due to the limitation of resources and time, only 86 patients were included in this study and the small number of participants may have affected the accuracy of the results. In addition, since many patients experience multiple chronic diseases, the surgical medication and anesthesia scheme are different, which may affect the accuracy of our data. Some subjective factors might impact the research results. In this study, we mainly explored the inhibitory effect of dexmedetomidine treatment on anesthesia-induced systemic inflammatory response before anesthesia induction. The time we took the blood sample was also during and shortly after the operation. Therefore, the regulatory effect of dexmedetomidine on inflammation in the postoperative recovery of patients undergoing intestinal surgery is still not fully understood. Furthermore, in this experiment, we only adopted a single intravenous infusion method and dosage. Different administration methods and dosages are likely to affect the effect of dexmedetomidine on the inflammatory response of patients. Therefore, in the following research, we will continue to pay attention to these problems and try to design some new experiments to solve these deficiencies in this research.

\section{Conclusion}

We mainly investigated the relationship between the application of dexmedetomidine during the intraoperative period and the outcomes of intestinal surgery patients in this research. We demonstrated that dexmedetomidine could effectively reduce the level of serum inflammatory factors in patients and inhibit the inflammation caused by intestinal 
surgery. Dexmedetomidine also alleviated the patient's stress response and promoted the recovery of the patient's cognitive ability. We believe that our research can provide a theoretical basis for the clinical application of dexmedetomidine and help to improve the prognosis and life quality of patients undergoing intestinal surgery. Nevertheless, this study was more focused on inflammation and the relative events. It is of great interest to also explore, in future studies, the effect of dexmedetomidine on gastrointestinal function recovery in patients undergoing intestinal surgery.

\section{Declarations}

Funding This study was supported by Quanzhou Science and Technology Project (2019N042S).

Conflict of interest Rushuang Chen, Zhenming Kang, Yaduan Wang, Jie Zhao, and Shunyuan Li declare no potential conflicts of interest with respect to the research, authorship, and/or publication of this article.

Ethics approval This study was approved by the Ethical Committee of Quanzhou First Hospital Affiliated to Fujian Medical University.

Consent to participate Written informed consent for publication of this paper was obtained from the Quanzhou First Hospital Affiliated to Fujian Medical University.

Consent for publication Written informed consent was obtained from patients for the publication of this study and any accompanying images. A copy of the written consent is available for review by the Editor-inChief of this journal.

Availability of data and material Data will be made available upon reasonable request.

Code availability Not applicable.

Author contributions RC, ZK, YW, JZ, SL conducted the experiments, collected and analyzed the data, and wrote the manuscript. SL conceived and supervised the study.

Open Access This article is licensed under a Creative Commons Attribution-NonCommercial 4.0 International License, which permits any non-commercial use, sharing, adaptation, distribution and reproduction in any medium or format, as long as you give appropriate credit to the original author(s) and the source, provide a link to the Creative Commons licence, and indicate if changes were made. The images or other third party material in this article are included in the article's Creative Commons licence, unless indicated otherwise in a credit line to the material. If material is not included in the article's Creative Commons licence and your intended use is not permitted by statutory regulation or exceeds the permitted use, you will need to obtain permission directly from the copyright holder. To view a copy of this licence, visit http://creativecommons.org/licenses/by-nc/4.0/.

\section{References}

1. Le Gall M, Thenet S, Aguanno D, Jarry AC, Genser L, RibeiroParenti L, et al. Intestinal plasticity in response to nutrition and gastrointestinal surgery. Nutr Rev. 2019;77(3):129-43. https://doi. org/10.1093/nutrit/nuy064.

2. Liu C, Wang ZZ, Su XQ. A decade's review for the foundation, establishment and development of the Department of Minimally Invasive Gastrointestinal Surgery in Peking University Cancer Hospital [in Chinese]. Zhonghua Wei Chang Wai Ke Za Zhi. 2019;22(8):719-23. https://doi.org/10.3760/cma.j.issn.1671-0274. 2019.08 .004$.

3. Wang Z, Chen J, Wang P, Jie Z, Jin W, Wang G, et al. Surgical site infection after gastrointestinal surgery in China: a multicenter prospective study. J Surg Res. 2019;240:206-18. https://doi.org/ 10.1016/j.jss.2019.03.017.

4. Sanchez de Medina F, Romero-Calvo I, Mascaraque C, MartinezAugustin O. Intestinal inflammation and mucosal barrier function. Inflamm Bowel Dis. 2014;20(12):2394-404. https://doi.org/10. 1097/MIB.0000000000000204.

5. Mitchell EL, Davis AT, Brass K, Dendinger M, Barner R, Gharaibeh $\mathrm{R}$, et al. Reduced intestinal motility, mucosal barrier function, and inflammation in aged monkeys. J Nutr Health Aging. 2017;21(4):354-61. https://doi.org/10.1007/s12603-016-0725-y.

6. Sauaia A, Moore FA, Moore EE. Postinjury inflammation and organ dysfunction. Crit Care Clin. 2017;33(1):167-91. https:// doi.org/10.1016/j.ccc.2016.08.006.

7. Stewart AG, Beart PM. Inflammation: maladies, models, mechanisms and molecules. Br J Pharmacol. 2016;173(4):631-4. https:// doi.org/10.1111/bph.13389.

8. Yu J-S, Jin J, Li Y-Y. The Physiological functions of IKK-selective substrate identification and their critical roles in diseases. STEMedicine. 2020;1(4): e49. https://doi.org/10.37175/stemedicine. v1i4.49.

9. Sottas CE, Anderson BJ. Dexmedetomidine: the new all-inone drug in paediatric anaesthesia? Curr Opin Anaesthesiol. 2017;30(4):441-51. https://doi.org/10.1097/ACO.0000000000 000488 .

10. Barends CR, Absalom A, van Minnen B, Vissink A, Visser A. Dexmedetomidine versus midazolam in procedural sedation. A systematic review of efficacy and safety. PLoS One. 2017;12(1):e0169525. doi: https://doi.org/10.1371/journal.pone. 0169525 .

11. Jun JH, Kim KN, Kim JY, Song SM. The effects of intranasal dexmedetomidine premedication in children: a systematic review and meta-analysis. Can J Anaesth. 2017;64(9):947-61. https://doi. org/10.1007/s12630-017-0917-x.

12. Li B, Li Y, Tian S, Wang H, Wu H, Zhang A, et al. Anti-inflammatory effects of perioperative dexmedetomidine administered as an adjunct to general anesthesia: a meta-analysis. Sci Rep. 2015;5:12342. https://doi.org/10.1038/srep12342.

13. Zhang DF, Su X, Meng ZT, Li HL, Wang DX, Xue-Ying L, et al. Impact of dexmedetomidine on long-term outcomes after noncardiac surgery in elderly: 3-year follow-up of a randomized controlled trial. Ann Surg. 2019;270(2):356-63. https://doi.org/10. 1097/SLA.0000000000002801.

14. Lin J, Li JB, Lu Z. Clinical application and effect of dexmedetomidine in combination with continuous positive airway pressure on one-lung ventilation in lung surgery of elder patients. Pak J Pharm Sci. 2018;31(6):2879-83.

15. Li M, Wang T, Xiao W, Zhao L, Yao D. Low-dose dexmedetomidine accelerates gastrointestinal function recovery in patients undergoing lumbar spinal fusion. Front Pharmacol. 2019;10:1509. https://doi.org/10.3389/fphar.2019.01509. 
16. Jiang L, Hu M, Lu Y, Cao Y, Chang Y, Dai Z. The protective effects of dexmedetomidine on ischemic brain injury: a meta-analysis. J Clin Anesth. 2017;40:25-32. https://doi.org/10.1016/j.jclin ane.2017.04.003.

17. Trivedi D. Cochrane review summary: mini-mental state examination (MMSE) for the detection of dementia in clinically unevaluated people aged 65 and over in community and primary care populations. Prim Health Care Res Dev. 2017;18(6):527-8. https:// doi.org/10.1017/S1463423617000202.

18. Pedziwiatr M, Mavrikis J, Witowski J, Adamos A, Major P, Nowakowski M, et al. Current status of enhanced recovery after surgery (ERAS) protocol in gastrointestinal surgery. Med Oncol. 2018;35(6):95. https://doi.org/10.1007/s12032-018-1153-0.

19. Friedell ML. The general surgery residency at Orlando Health: past, present, and future. Am Surg. 2010;76(1):7-10.

20. Potts JR 3rd. General surgery residency: Past, present and future. Curr Probl Surg. 2019;56(5):174-97. https://doi.org/10.1067/j. cpsurg.2019.01.005

21. Han G, Qiao X, Ma Z. Diagnosis and treatment of intra-abdominal infection complicated with hypothyroidism [in Chinese]. Zhonghua Wei Chang Wai Ke Za Zhi. 2018;21(12):1356-60.

22. Liu H, Zhou Z. Management strategy of intra-abdominal infection caused by multidrug-resistant bacteria [in Chinese]. Zhonghua Wei Chang Wai Ke Za Zhi. 2018;21(12):1351-5.

23. Menna C, De Falco E, Teodonio L, Andreetti C, Maurizi G, Ciccone AM, et al. Surgical wound-site inflammation: video-assisted thoracic surgery versus thoracotomy. Interact Cardiovasc Thorac Surg. 2019;28(2):240-6. https://doi.org/10.1093/icvts/ivy231.

24. Shiroky J, Lillie E, Muaddi H, Sevigny M, Choi WJ, Karanicolas PJ. The impact of negative pressure wound therapy for closed surgical incisions on surgical site infection: a systematic review and meta-analysis. Surgery. 2020;167(6):1001-9. https://doi.org/ 10.1016/j.surg.2020.01.018.

25. Hyldig N, Birke-Sorensen H, Kruse M, Vinter C, Joergensen JS, Sorensen JA, et al. Meta-analysis of negative-pressure wound therapy for closed surgical incisions. Br J Surg. 2016;103(5):47786. https://doi.org/10.1002/bjs.10084.

26. Gabriel A, Gupta S, Orgill DP. Challenges and Management of Surgical Site Occurrences. Plast Reconstr Surg. 2019;143(1):7S10S. https://doi.org/10.1097/PRS.0000000000005305 (1S Management of Surgical Incisions Utilizing Closed-Incision Negative-Pressure Therapy).

27. Villela NR, Nascimento JP. Dexmedetomidine in anesthesiology [in Portuguese]. Rev Bras Anestesiol. 2003;53(1):97-113. https:// doi.org/10.1590/s0034-70942003000100013.

28. Kulikov AS, Lubnin AY. Dexmedetomidine: new opportunities in anesthesiology [in Russian]. Anesteziol Reanimatol. 2013;1:37-41.

29. Flanders CA, Rocke AS, Edwardson SA, Baillie JK, Walsh TS The effect of dexmedetomidine and clonidine on the inflammatory response in critical illness: a systematic review of animal and human studies. Crit Care. 2019;23(1):402. https://doi.org/10. 1186/s13054-019-2690-4.

30. Cozzi G, Norbedo S, Barbi E. Intranasal dexmedetomidine for procedural sedation in children, a suitable alternative to chloral hydrate. Paediatr Drugs. 2017;19(2):107-11. https://doi.org/10. 1007/s40272-017-0217-5.

31. Weerink MAS, Struys M, Hannivoort LN, Barends CRM, Absalom AR, Colin P. Clinical pharmacokinetics and pharmacodynamics of dexmedetomidine. Clin Pharmacokinet. 2017;56(8):893913. https://doi.org/10.1007/s40262-017-0507-7.

32. Rajan S, Talukdar R, Tosh P, Paul J, Vasu BK, Kumar L. Hemodynamic responses and safety of sedation following premedication with dexmedetomidine and fentanyl during fiberoptic-assisted intubation in patients with predicted difficult airway. Anesth Essays Res. 2018;12(1):11-5. https://doi.org/10.4103/aer.AER_176_17.

33. Jaideep CN, Bhargava DV. Good vagal tone, a tourniquet and dexmedetomidine: recipe for disaster. Indian J Anaesth. 2015;59(7):450-1. https://doi.org/10.4103/0019-5049.160965. 